\title{
Field study on soil collapsibility sensitivity
}

\author{
Yao Zhang ${ }^{1}$, Shaoqiang $\mathrm{Guo}^{1}$, Ning $\mathrm{Li}^{2}$, Zaiqiang $\mathrm{Hu}^{2, \mathrm{a}}$, Zhixiang $\mathrm{Zhao}^{3}$, Yu Xi ${ }^{1}$, Shuai Shao ${ }^{2}$ \\ ${ }^{1}$ Shaanxi Key Laboratory of Safety and Durability of Concrete Structures, Xijing University, Xi'an 710123, China. \\ ${ }^{2}$ School of Civil Engineering and Architecture, Xi'an University of Technology, Xi' an 710048, China. \\ ${ }^{3}$ Northwest Engineering Corporation Limited, Xi' an 710065, China.
}

\begin{abstract}
The sensitivity of loess collapsibility is generally considered as the difficulty, speed and size of loess collapsibility after water immersion. It is different from collapsibility of loess, but they are related to each other. In this paper, the time to complete $90 \%$ collapsibility and the coefficient of collapsibility are used to evaluate the collapsibility sensitivity of loess and its site. The method is verified by taking the site with excessive collapsibility as an example. The results show that the method can accurately reflect the collapsibility sensitivity of collapsible site, and has certain guiding significance for engineering construction on collapsible site. In addition, the method is simple, scientific, and can be combined with the current specifications, so it is easy to be accepted and promoted by the industry, and is a useful supplement to the existing few researches on loess collapsibility sensitivity.
\end{abstract}

\section{Introduction}

The fundamental reason of loess collapsibility lies in its unique internal material composition and structural characteristics. Microscopic observation shows that loess is composed of structural units (single ore body, aggregate and agglomerate), cements (clay, organic matter and calcium carbonate) and pores (macropores, overhead pores and intergranular pores). When the loess is infiltrated by water, the strength of salt crystal cementation will be weakened, and it will lose stability under a certain pressure, resulting in other micro structural units around the pores falling into the pores [1] [2] [3]. Therefore, salt crystal cementation (including insoluble salt, moderately soluble salt and easily soluble salt) plays an important role in loess collapsibility, which largely determines the difficulty, speed and size of loess collapsibility, that is, the collapsibility sensitivity of loess.

Because salt crystal cementation belongs to microstructure, it is difficult to test its mechanical properties by conventional mechanical tests. Therefore, there are few experimental studies on the effect of salt crystal cementation on loess collapsibility and collapsibility sensitivity at home and abroad. In order to understand the mechanism of salt crystal cementation in loess collapsibility and collapsibility sensitivity. The author uses the characteristic that nitric acid can rapidly dissolve salt crystal cementation, adds different concentrations of nitric acid into the immersion solution of loess collapsibility test, and tests the collapsibility sensitivity indexes of loess, such as collapsibility coefficient, stability time and collapsibility rate, under different acidic environments and different pressures ${ }^{[4]}{ }^{[5]}$.

In contrast with the conventional collapsibility test, the change and mechanism of cemented connection in collapsibility and collapsibility sensitivity of loess are reflected. In addition, in order to further promote the construction of geotechnical engineering in collapsible loess area, another important purpose of this paper is to interpret the characteristics of loess collapsibility sensitivity based on some data of this test, and on this basis, combined with the experience of predecessors, put forward a scientific and fair method which can be closely linked with the current specification, It is easy to be accepted and popularized to evaluate the collapsibility sensitivity of loess.

\section{Evaluation method}

\subsection{Evaluation of collapsibility sensitivity of soil samples}

The author puts forward that $\mathrm{t}$ (the time needed to complete $90 \%$ of the total amount of collapsibility) is divided according to the speed of occurrence and the probability of occurrence, and the collapsibility coefficient of loess is directly divided by the method of 4.2.2 in the building code for collapsible loess area. Finally, the collapsibility sensitivity is determined by these two elements, which can be divided into four grades according to the order from weak to strong. The evaluation method is based on the definition or essential meaning of collapsibility sensitivity and belongs to comprehensive index evaluation method. Its scientificity and practicability will be verified in the section of 4.3 collapsibility sensitivity evaluation example. 3 Evaluation of collapsibility sensitivity of Loess ${ }^{[6]}[7]$.

\footnotetext{
${ }^{\mathrm{a}}$ Corresponding author: 2596219750@qq.com
} 
Table 1. Grades of collapse sensitivity in loess

\begin{tabular}{cccc}
\hline $\begin{array}{c}I_{c}(\%) \\
\mathrm{T} \text { (min) }\end{array}$ & $0.015<I_{c} \leq 0.03$ & $0.03<I_{c} \leq 0.07$ & $I_{c}>0.07$ \\
\hline $60<T$ & Slight & Slight & Moderate \\
$15<T \leq 60$ & Slight & Moderate & Moderately severe \\
$5 \leq T \leq 15$ & Moderate & Moderately severe & Severe \\
$T<5$ & Moderately severe & Severe & Severe \\
\hline
\end{tabular}

\subsection{Evaluation of collapsibility sensitivity of loess site}

In situ site, the collapsibility sensitivity is also affected by the thickness and permeability coefficient of different soil layers, and the above judgment method of loess collapsibility sensitivity can only reflect the collapsibility sensitivity of a certain part or a certain layer of excavated soil. The collapsibility sensitivity of the site should be evaluated in at least three representative sites on the site plane. When the site soil is uneven, it should be increased appropriately. For each selected representative site, one soil sample should be taken from the shallow soil (less than or equal to $6 \mathrm{~m}$ ) and the deep soil (more than $6 \mathrm{~m}$ ). When the buried depth of collapsible loess in the site is large, the number of sampling layers should be appropriately increased. For the sampling method, please refer to the schematic diagram of selecting sampling points in Figure 6 . The collapsibility sensitivity grade of the site should be the highest in the soil samples taken from the site. Because the soil layers of the same site may have large difference in collapsibility sensitivity. As long as there is one or one layer of soil in the site with high collapsibility sensitivity, the whole site may be harmed by the corresponding collapsibility, or even by the uneven settlement.

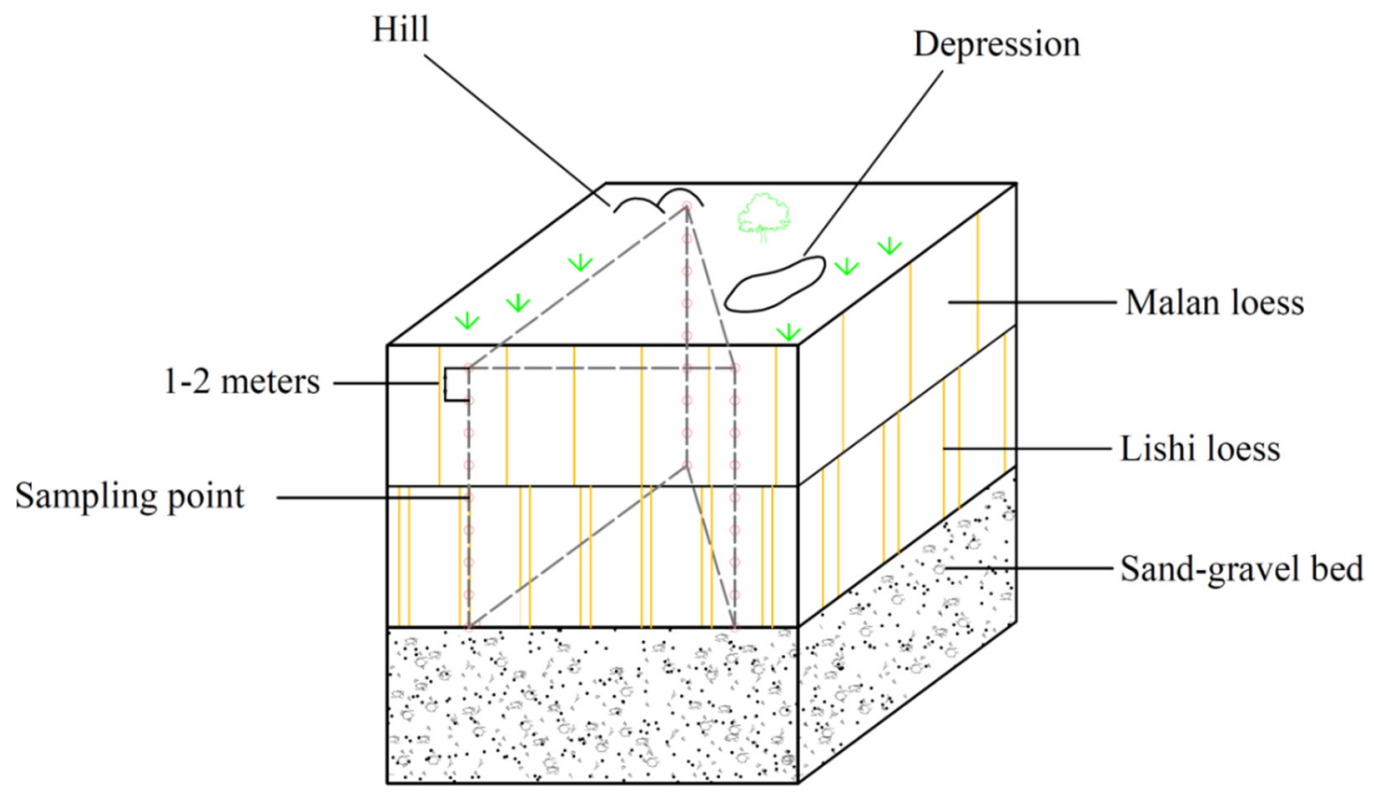

Figure 1. Diagram of selected sampling points

\section{Collapsibility sensitivity evaluation}

\subsection{Example of collapsibility sensitivity evaluation}

In this paper, the collapsible ground fissures in the south side of the drainage canal in dongtaifu village, Liangyi Town, Dali County, Shaanxi Province are taken as an example to verify the evaluation method ${ }^{[7]}$. According to the above method, six soil samples of exploration wells tj4, $\mathrm{tj} 5$ and $\mathrm{tj} 6$ in $4.5 \mathrm{~m}$ and $7.5 \mathrm{~m}$ layers are selected for this verification, and their collapsibility sensitivity indexes are shown in Table 5. On the basis of the plateau, the collapsibility sensitivity of the site is determined to be very strong. According to the survey report, a total of 9 collapsible ground fissures were found in the survey area, the longest of which is $72 \mathrm{~m}$ and the width is $5-20 \mathrm{~cm}$, causing serious damage to many local buildings, such as houses, roads and canals. The actual situation is consistent 
with the evaluation results of this method.

Cui yue'e suggested that the settlement rate of $5 \mathrm{~min}$ should be used to determine the collapsibility sensitivity of loess [5]. According to her evaluation method, the average settlement rate of $5 \mathrm{~min}$ of the site soil sample is $0.216 \mathrm{~mm}$, and the sensitivity level is high. Compared with this method, the method proposed in this paper considers the possible change of collapsibility sensitivity after 5 minutes, and has a wide range of time surface, and has a comprehensive evaluation on the soil samples of the whole site. The evaluation method proposed by some other scholars ${ }^{[6]}$ is not widely used because of its early age, so this paper will not discuss it.

Table 2. Collapse sensitivity of loess at Chou Huang ditch

\begin{tabular}{cccccc}
\hline \multirow{2}{*}{ No } & \multicolumn{3}{c}{ Collapsible deformation $(0.001 \mathrm{~mm}) /$} & \multirow{2}{*}{$\begin{array}{c}\text { Stability } \\
\text { Percentage }\end{array}$} & \multirow{2}{*}{$I_{c}$} \\
\cline { 2 - 4 } & $5 \mathrm{~min}$ & $30 \mathrm{~min}$ & Stability & & \\
\cline { 2 - 5 } 1 & $200 / 0.44$ & $350 / 0.77$ & $455 / 1$ & 4.5 & 0.023 \\
2 & $600 / 0.61$ & $874 / 0.88$ & $990 / 1$ & 4 & 0.050 \\
3 & $105 / 0.13$ & $280 / 0.88$ & $838 / 1$ & 7 & 0.042 \\
4 & $220 / 0.5$ & $393 / 0.9$ & $436 / 1$ & 2.5 & 0.022 \\
5 & $300 / 0.38$ & $348 / 0.44$ & $796 / 1$ & 4 & 0.040 \\
6 & $180 / 0.58$ & $302 / 0.97$ & $312 / 1$ & 1.5 & 0.016 \\
7 & $285 / 0.19$ & $403 / 0.27$ & $1506 / 1$ & 8 & 0.075 \\
8 & $825 / 0.46$ & $1315 / 0.73$ & $1800 / 1$ & 6 & 0.09 \\
9 & $78 / 0.24$ & $168 / 0.51$ & $335 / 1$ & 4.5 & 0.017 \\
10 & $496 / 0.71$ & $688 / 0.99$ & $697 / 1$ & 1.5 & 0.035 \\
11 & $1080 / 0.87$ & $1176 / 0.95$ & $1243 / 1$ & 4 & 0.057 \\
12 & $1328 / 0.85$ & $1437 / 0.92$ & $1562 / 1$ & 2.5 & 0.078 \\
13 & $227 / 0.64$ & $288 / 0.81$ & $355 / 1$ & 2.9 & 0.018 \\
14 & $558 / 0.45$ & $830 / 0.67$ & $1240 / 1$ & 6.5 & 0.062 \\
& & & & & \\
\hline
\end{tabular}

\subsection{Description of evaluation method}

In Figure 1, the collapsibility coefficients of soil samples under distilled water solution at $200 \mathrm{kPa}$ and $800 \mathrm{kPa}$ are about 0.06 . However, it can be seen from Figure 2 that the stability time of the three solutions varies greatly at $1600 \mathrm{kpa}, 184,214$ and $394 \mathrm{~min}$ respectively. It is very small that there is such a big difference in the acidity of the immersion solution on the site, but the same situation will occur when the water solution is immersed in different soil samples ${ }^{[7]}$. Therefore, collapsibility sensitivity is an important characteristic of loess collapsibility. In this paper, the two indexes of collapsibility sensitivity, collapsibility rate and collapsibility deformation, are described respectively.

In this paper, many forms of collapsibility sensitivity are simplified as the speed of collapsibility rate and the amount of collapsible deformation. The time to complete $90 \%$ of the total amount of collapsibility and the collapsibility coefficient are selected as the evaluation indexes of this method. This method belongs to the index comprehensive evaluation method, can be specific to the evaluation of a site, and has guiding significance for specific projects. This method is combined with the current specifications, with few evaluation parameters and simple test instruments, so it is easy to be accepted and promoted by the industry. Based on the previous experience and experimental data, the evaluation method of collapsibility sensitivity of loess is proposed. Although this method has been determined, due to the limited data collected, the division of $\mathrm{T}$ (time to complete $90 \%$ collapsible volume) may not be perfect. In the future, it may be necessary for the majority of colleagues to improve the division of this parameter. In addition, the division basis of this paper mainly comes from the collapsibility test of water solution, and the main purpose is to evaluate the sensitivity of loess collapsibility under natural conditions, so this method is suitable for the collapsibility test of ordinary water solution, not for the evaluation of unconventional solution.

\section{Conclusion}

In this paper, the effect of cemented connection in Loess on Collapsibility and collapsibility sensitivity is revealed through the difference between acid collapsibility test and ordinary collapsibility test. It is the first time to evaluate the collapsibility sensitivity of loess and its site by using two indexes of time and collapsibility coefficient to complete $90 \%$ of the total collapsibility. The main conclusions are as follows

Based on the interpretation of the definition of collapsibility sensitivity and the analysis of the test data, it is proposed for the first time to evaluate the collapsibility sensitivity of Loess by using two parameters: collapsibility coefficient and time to complete $90 \%$ of the total collapsibility. According to the collected data of collapsibility tests and the requirements of "building code for collapsible loess area", the collapsibility sensitivity of loess is divided into four grades: weak, medium, strong and very strong. Based on the division of loess collapsibility sensitivity, the evaluation of site 
collapsibility sensitivity is put forward, that is, according to the "higher" principle of loess collapsibility sensitivity at sampling points. Finally, a case study of the Yellow River drainage channel, which has experienced collapsibility, shows that the method is simple, scientific and practical.

\section{Acknowledgements}

This work was supported by the China Postdoctoral Science Foundation (Grant No. 2020M673617XB), the Open Research Fund of State Key Laboratory of Geomechanics and Geotechnical Engineering, Institute of Rock and Soil Mechanics, Chinese Academy of Sciences (Grant No. Z020019), the special Foundation for High Level Talents of Xijing university (Grant No. XJ20B12).

\section{Reference:}

1. Rao, S. M., Revanasiddappa, K. Collapse behaviour of a residual soil [J]. Geotechnique. 2002, 52 (52): 259-268.

2. Hu Zai-qiang, Shen Zhu-jiang, Xie Ding-yi. Deformation properties of structural loess [J]. Chinese Journal of Rock Mechanics and Engineering, 2004, 23(24): 4142-4146

3. Hu Zai-qiang, Shen Zhu-jiang, Xie Ding-yi. The structure of unsaturated loess [J]. Chinese Journal of Rock Mechanics and Engineering, 2000, 19(06): 775779

4. Lawton EC, Fragaszy RJ, and Hetherington MD (1992) Review of Wetting-Induced Collapse in Compacted Soil. Journal of Geotechnical Engineering 118 (9): 1376-1394.

5. Lutenegger AJ (2012) Collapse "Sensitivity" of Midcontinent and Lower Mississippi Valley Loess. Geocongress 225: 880-889.

6. Lv QF, Wang SF, Wang DK, and Wu Z (2014) Water stability mechanism of silicification grouted loess. Bulletin of Engineering Geology and the Environment 73 (4): 1025-1035.

7. Zhang $\mathrm{Y}, \mathrm{Hu}$ ZQ, Chen $\mathrm{H}$, Xue $\mathrm{T}$ (2018) Experimental investigation of the behavior of collapsible loess treated with the acid-addition presoaking method. KSCE Journal of civil engineering. 